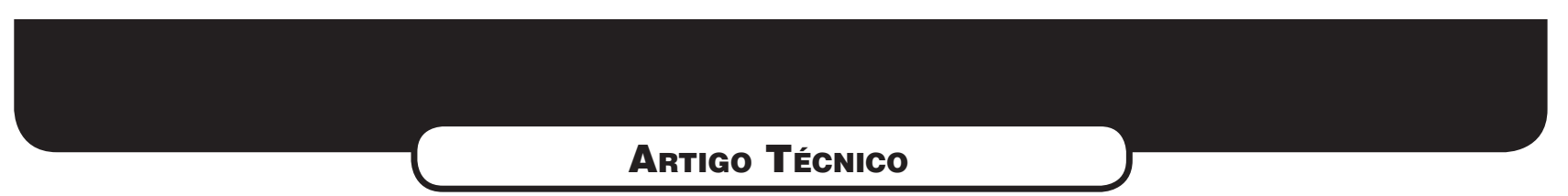

\title{
TESTES DE TOXICIDADE AGUDA ATRAVÉS DE BIOENSAIOS NO EXTRATO SOLUBILIZADO DOS RESÍDUOS CLASSE II A - NÃO INERTES E CLASSE II B - INERTES
}

\author{
ACUTE TOXICITY TESTS BY BIOASSAYS APPLIED TO THE SOLUBILIZED EXTRACTS \\ OF SOLID WASTES CLASS II A - NON INERTS AND CLASS II B - INERTS
}

\begin{abstract}
NÉBORA LIZ VENDRAMIN BRASIL RODRIGUES
Engenheira Química pela PUC-PR. Mestre em Engenharia de Recursos Hídricos e Ambiental - Área de Engenharia Ambiental (UFPR)

\section{URIVALD PAWLOWSKY}

Professor Titular dos cursos de pós-graduação em Engenharia de Recursos Hídricos e Ambiental, Tecnologia Química e Engenharia Química da UFPR. Mestre em Ciência pela COPPE - UFRJ. Doutor pela State University of New York.

Consultor da Organização Pan-Americana de Saúde, de Indústrias e de Orgãos Governamentais
\end{abstract}

Recebido: 19/10/05 Aceito: 04/10/06

\section{RESUMO}

A grande diversidade de substâncias potencialmente tóxicas contribuem para a deterioração do meio ambiente. O objetivo deste trabalho foi propor a utilização de bioensaios, através de testes de toxicidade aguda com Daphnia magna e Vibrio fischeri, como mais um parâmetro a ser analisado no extrato solubilizado dos resíduos que, segundo a NBR 10004/04 fossem classificados como classe II A - não inertes ou classe II B - inertes. Realizaram-se, também, testes de toxicidade no drenado dos aterros classe II A e II B. Verificou-se que a toxicidade foi constatada nos extratos solubilizados dos 18 resíduos analisados e que, apenas três das amostras estariam próprias para lançamento, ou seja os resíduos 04, 14 e 15. Já, a toxicidade encontrada no drenado dos aterros, ficou muito superior do que a toxicidade de cada extrato solubilizado analisado separadamente.

PALAVRAS-CHAVE: Resíduo slido, lixiviação, solubilização, bioensaios, NBR 10004/04 classificação de resíduos.

\begin{abstract}
A great diversity of substances potencially toxic contributes to the deterioration of the environment. The aim of this research was to propose the use of bioassays using Daphnia magna and Vibrio fischeri, as another parameter to be analyzed in the solubilized extraction of waste according to NBR 10004/04 and classified as class II $A$ - non inerts or class II B-inerts. Besides, another test was performed to measure the level of toxicity in the drainage of the landfill class II A and II B. It was verified that the toxicity found in the solubilized extracts of the 18 wastes analysed. Only 3 wastes (04, 14 and 15) were within the emission limits. On the other hand the toxicity found in the drainage of the landfill, from which all the samples came from, was much higher than the individual one.
\end{abstract}

KEYWORDS: Solid waste, leaching, solubilization, bioassays, bioindicators, NBR 10004/04 waste classification.

\section{INTRODUÇÃO}

Entre as diversas formas de poluição ambiental está a poluição por resíduos industriais, onde apresentamse graves problemas sócio econômicos e ambientais. Envolvem-se nesse problema a geração, o acondicionamento, o manuseio, a coleta, o transporte, o tratamento e a disposição final (Jacomino et al, 2002).

A contaminação do solo e das águas podem ocorrer quando a disposição final de resíduos é feita de maneira inadequada, possibilitando que poluentes afetem o meio ambiente quando arrastados com a água ou solubilizados. (Zulauf, 1977). O aterramento é considerado uma alternativa para a disposição final de resíduos sólidos industriais, sendo que para esta alternativa deve ser recomendado que os resíduos estejam estáveis e com baixo teor de umidade (Jacomino et al, 2002).

A Norma NBR 10004 (ABNT, 2004a) classifica os resíduos quanto à sua periculosidade ao meio ambiente e à saúde pública e indica a destinação adequada para estes resíduos. Para a aplicação desta norma, é necessário consultar as Normas Complementares das quais fazem parte a lixiviação, so- lubilização e amostragem de resíduos. Alguns resíduos são de difícil classificação e até mesmo os já classificados e titulados como classe II A e II B, ainda que submetidos ao teste de solubilização conforme a NBR 10006 (ABNT, 2004c), não garantem ausência de toxicidade.

A toxicidade é uma propriedade inerente à substância que produz efeitos danosos aos organismos expostos, durante um determinado tempo, a uma concentração específica (ABNT, 2004e). $\mathrm{O}$ efeito agudo se manifesta dentro de períodos curtos (horas ou dias) de exposição dos organismos aquáticos a um 
agente nocivo, causando quase sempre a letalidade. Pode também ocorrer a imobilidade à alguns microcrustáceos (Knie e Lopes, 2004).

Uma das formas de entrada de agentes tóxicos para o ambiente aquático se dá através do resíduo sólido industrial. Não se deve subestimar o controle de poluentes tóxicos que afetam adversamente o meio ambiente, devido à grande diversidade de novas substâncias que estão sendo introduzidas no mesmo (Zagatto et al, 1992). Ainda, a caracterização química de um efluente não corresponde ao potencial tóxico de uma mistura de contaminantes (Cardoso e Luca, 2004). Desse modo ocorre uma maior complexidade quando se pretende estimar os danos que uma substância específica pode causar aos organismos e identificar, com exatidão, qual agente tóxico é o causador do desequilíbrio (CETESB, 1990b). A avaliação da toxicidade mediante bioensaios com organismos indicadores, tais como fotobactérias e daphnias, fornece um parâmetro somatório dos teores de diversos poluentes tóxicos. Assim, não basta reduzir a carga de uma única substância em um efluente para que não ocorra efeito tóxico aos organismos (Knie e Lopes, 2004). A maioria dos agentes químicos, principalmente os perigosos, são acumulativos no meio aquático, portanto, a biota aquática pode estar sujeita aos efeitos dessas substâncias por prolongados períodos de tempo (FATMA, 1999).

O objetivo deste trabalho foi propor a introdução de bioensaios na NBR 10004/04, como testes complementares, para a avaliação da toxicidade aguda do extrato solubilizado dos resíduos sólidos industriais enquadrados como classe II A - não inertes e classe II $\mathrm{B}$ - inertes.

Para atingir o objetivo principal, o presente estudo selecionou resíduos pré-classificados como classe II A - não inertes e classe II B - inertes, e os submeteu a ensaios de solubilização. A partir do solubilizado foram realizados bioensaios utilizando Daphnia magna e Vibrio fischeri, como organismos bioindicadores de toxicidade, possibilitando avaliar a toxicidade do resíduo sólido industrial.

A importância do presente trabalho está justamente em mostrar o nível tóxico do solubilizado dos resíduos de diversos ramos industriais para que assim seja avaliada a eficiência da aplicação da NBR 10004/04 - Classificação de resíduos, para evitar efeitos tóxicos nos corpos d'água causados pelos drenados dos aterros. E, desta forma, verificar a necessidade de se agregar parâmetros biológicos, os quais permitem conhecer o nível tóxico do extrato solubilizado de um determinado resíduo, na avaliação dos resíduos para seu enquadramento nas classes previstas.

\section{MATERIAIS E MÉTODOS}

Foram testadas dezoito amostras de resíduos pré-classificados, sendo dezessete como classe II A- não inertes e uma como classe II B- inertes. Também, quatro amostras de drenado dos aterros de resíduos classe II A e classe II $\mathrm{B}$ foram submetidas à análises. As amostras foram cedidas por uma unidade de disposição final de resíduos, onde a destinação final é de acordo com a tipologia e classificação dos resíduos, conforme descritos na Tabela 1. A classificação destes resíduos foi realizada antes de entrar em vigor a NBR 10004/04, então, as classes foram nomeadas como classe II ou IIA e III ou IIB.

Os drenados analisados foram coletados dos aterros em datas e condiçôes do tempo variadas, sendo que somente na primeira coleta o dia apresentava-se chuvoso.

Foram realizados testes de lixiviação nos resíduos 11, 12, 13, 14 e 15, escolhidos aleatoriamente, de acordo com a NBR 10005 (ABNT, 1987b). Os testes de solubilização foram aplicados nos 18 resíduos, de acordo com a NBR 10006 (ABNT, 1987c). Todos os testes foram executados nos Laboratórios de Tecnologia Ambiental do Instituto Ambiental do Paraná.

Somente cinco amostras foram submetidas ao ensaio de lixiviação devido ao custo elevado das análises e ao fato de que os resíduos já estavam pré-classificados como classe II A e classe II B.

Tanto os extratos lixiviado quanto os solubilizado, foram submetidos a determinação de metais pelo Laboratório de Absorção Atômica do IAP, com o intuito de comparação de resultados de classificação obtidos por dois laboratórios diferentes. A técnica aplicável para a determinação de metais foi Espectrofotometria de Absorção Atômica em Chama de Ar-Acetileno (APHA, 1998).

Os testes de toxicidade, foram aplicados nas dezoito amostras de solubilizado, assim como nas quatro amostras de drenados dos aterros, e desenvolvidos no Laboratório de Ecotoxicologia do IAP, sendo o microcrustáceo Daphnia magna e a bactéria Vibrio fischeri os organismos bioindicadores de toxicidade utilizados, pois estes são considerados como padrão internacional para testes de toxicidade aguda (Knie e Lopes, 2004). O método utilizado para a avaliação da toxicidade aguda com Daphnia magna foi de acordo com a ABNT (2004e), a qual é baseada na ISO 6341(E) e DIN 38412(30)(11). Já o método utilizado para a avaliação da toxicidade aguda com Vibrio fischeri foi de acordo com o Manual de Métodos para Avaliação da Toxicidade (IAP, 1997), o qual é baseado em normas da ISO 11348-1 e do DIN 38412-34.

Os resultados dos efeitos observados, tanto para a $D$. magna quanto para $V$. fischeri, foram expressos em fator de toxicidade (FT), onde este representa para o microcrustáceo D. magna, o menor valor de diluição da amostra na qual não se observa imobilidade maior que $10 \%$ nos organismos expostos, e deve ser determinado através da observação direta da mobilidade dos organismos. Ainda, segundo Knie e Lopes (2004), para as bactérias $V$. fischeri, o FT é a primeira diluição onde a porcentagem de inibição da luminescência é inferior a 20\%. O fator de toxicidade (FT) é representado por um número inteiro, o qual corresponde a menor diluição da amostra que não cause efeito tóxico aos organismos (ABNT, 2004e).

\section{RESULTADOS E DISCUSSÃO}

Os resultados foram comparados com os limites estabelecidos pelas NBR's de 1987 e de 2004, sendo que a nominação das classes nas tabelas 2, 3, 5 e 6 foi mantida a da NBR 10004/87 (classe II e classe III), e na discussão destas tabelas foi utilizada a nominação das classes conforme a NBR 10004/04 (classe II A e classe II B).

O ensaio de lixiviação das amostras $11,12,13,14$ e 15 , foi realizado para comprovar que nenhum resíduo classificado como perigoso, estivesse entre os resíduos submetidos a bioensaios, e ainda, para comparar os resultados com o laudo de classificação de cada resíduo, elaborado pela unidade de destinação de resíduos. Na Tabela 2 são apresentados os resultados das concentrações de metais encontrados no extrato lixiviado. 
Tabela I - Lista das amostras cedidas pela unidade de destinação final, classificação conforme a NBR I0004/87, NBR I0004/04 e origem dos resíduos

\begin{tabular}{|c|c|c|c|}
\hline $\begin{array}{l}\text { Resíduo } \\
n^{\circ}\end{array}$ & $\begin{array}{c}\text { NBR } \\
10004 / 87\end{array}$ & $\begin{array}{c}\text { NBR } \\
10004 / 04\end{array}$ & Origem \\
\hline 01 & Classe II & Classe IIA & $\begin{array}{l}\text { Borra de fosfato. Lodo proveniente da estação de tratamento } \\
\text { de efluentes de um processo de fosfatização. }\end{array}$ \\
\hline 02 & Classe II & Classe IIA & $\begin{array}{l}\text { Lodo biológico oriundo do tratamento de efluentes do } \\
\text { processo de curtição de couro. }\end{array}$ \\
\hline 03 & Classe II & Classe IIA & $\begin{array}{l}\text { Lodo de estação de tratamento de efluentes proveniente do } \\
\text { processo de saponificação e neutralização. }\end{array}$ \\
\hline 04 & Classe II & Classe IIA & Lodo galvânico oriundo de processo de galvanoplastia. \\
\hline 05 & Classe II & Classe IIA & $\begin{array}{c}\text { Lodo de estação de tratamento de efluentes gerado na } \\
\text { indústria de alimentos. }\end{array}$ \\
\hline 06 & Classe II & Classe IIA & $\begin{array}{l}\text { Lodo de estação de tratamento de efluentes vindo do processo } \\
\text { de tingimento de fios de poliéster com corantes à base de água. }\end{array}$ \\
\hline 07 & Classe II & Classe IIA & $\begin{array}{c}\text { Terra e areia contaminada com óleo proveniente da lavagem de } \\
\text { equipamentos de terraplanagem. }\end{array}$ \\
\hline 08 & Classe III & Classe IIB & $\begin{array}{c}\text { Lodo de estação de tratamento de efluentes gerado na } \\
\text { indústria têxtil. }\end{array}$ \\
\hline 09 & Classe II & Classe IIA & Lodo de rampa de lavagem de veículos. \\
\hline 10 & Classe II & Classe IIA & $\begin{array}{l}\text { Lodo de estação de tratamento de efluentes gerados na } \\
\text { produção de cosméticos. }\end{array}$ \\
\hline 11 & Classe II & Classe IIA & $\begin{array}{l}\text { Resina endurecida originada das sobras do processo de } \\
\text { fabricação de resinas para impregnação de papel. }\end{array}$ \\
\hline 12 & Classe II & Classe IIA & Resíduo originado no processo de carbonização de pinus spp. \\
\hline 13 & Classe II & Classe IIA & $\begin{array}{l}\text { Lodo de estação de tratamento de efluentes do processo de } \\
\text { obtenção de resina de nó de pinho. }\end{array}$ \\
\hline 14 & Classe II & Classe IIA & Escória de siderúrgica coletado no lastro da estrada de ferro. \\
\hline 15 & Classe II & Classe IIA & Escória de siderúrgica coletado no pátio de escória. \\
\hline 16 & Classe II & Classe IIA & Lodo de estação de tratamento de efluentes de cervejaria. \\
\hline 17 & Classe II & Classe IIA & Terra infusória, oriunda do processo de filtração de cerveja. \\
\hline 18 & Classe II & Classe IIA & $\begin{array}{l}\text { Lodo do tratamento de efluente gerado na pintura dos assentos } \\
\text { de automóveis. }\end{array}$ \\
\hline
\end{tabular}

Fonte:Laudo de classificação da unidade de destinação final de resíduos

Tabela 2 - Resultados das análises de metais no extrato lixiviado

\begin{tabular}{|c|c|c|c|c|c|c|c|c|c|c|c|}
\hline \multirow[t]{2}{*}{ Referências } & \multirow[t]{2}{*}{ Classe } & \multicolumn{10}{|c|}{ Metais $(\mathrm{mg} / \mathrm{L})$} \\
\hline & & $\mathrm{Al}$ & $\mathrm{Ba}$ & $\mathrm{Cd}$ & $\mathrm{Cu}$ & $\mathrm{Cr}$ & $\mathrm{Fe}$ & Mn & $\mathrm{Na}$ & $\mathrm{Pb}$ & $\mathrm{Zn}$ \\
\hline $\begin{array}{l}\text { NBR10004/2004 } \\
\text { (Limite máximo) }\end{array}$ & IIA ou IIB & $\mathrm{NE}$ & 70,0 & 0,5 & $\mathrm{NE}$ & 5,0 & $\mathrm{NE}$ & $\mathrm{NE}$ & $\mathrm{NE}$ & 1,0 & $\mathrm{NE}$ \\
\hline $\begin{array}{l}\text { NBR10004/1987 } \\
\text { (Limite máximo) }\end{array}$ & II ou III & $\mathrm{NE}$ & 100,0 & 0,5 & $\mathrm{NE}$ & 5,0 & $\mathrm{NE}$ & $\mathrm{NE}$ & $\mathrm{NE}$ & 5,0 & $\mathrm{NE}$ \\
\hline $\begin{array}{l}\text { Limite de } \\
\text { Detecção }\end{array}$ & $\mathrm{NE}$ & 0,40 & 0,60 & 0,01 & 0,03 & 0,10 & 0,06 & 0,03 & 0,01 & 0,04 & 0,10 \\
\hline Resíduo 11 & II ou III & $<\mathrm{LD}$ & NA & NA & 0,08 & $<\mathrm{LD}$ & 0,28 & 0,05 & 6,66 & 0,06 & 0,31 \\
\hline Resíduo 12 & II ou III & $<\mathrm{LD}$ & NA & NA & 0,17 & $<\mathrm{LD}$ & 0,07 & 25,90 & 4,64 & 0,13 & 0,22 \\
\hline Resíduo 13 & II ou III & 0,40 & NA & NA & $<\mathrm{LD}$ & $<\mathrm{LD}$ & 0,23 & 0,68 & 5,70 & 0,05 & 0,42 \\
\hline Resíduo 14 & II ou III & NA & 3,30 & $<\mathrm{LD}$ & NA & $<\mathrm{LD}$ & NA & NA & NA & 0,04 & NA \\
\hline Resíduo 15 & II ou III & NA & 3,46 & $<\mathrm{LD}$ & NA & $<\mathrm{LD}$ & NA & NA & NA & $<\mathrm{LD}$ & NA \\
\hline
\end{tabular}

NE: não existente; NA: não analisado; LD: limite de detecção do método analítico utilizado; NBR 10004/04: classe IIa - não inertes e classe IIB - inertes; NBR 10004/87: classe II - não inertes e classe III - inertes 
Verificou-se através da Tabela 2, que os resíduos $11(0,08 \mathrm{mg} / \mathrm{L}$ de $\mathrm{Cu}, 0,28 \mathrm{mg} / \mathrm{L}$ de Fe, 0,05 mg/ $\mathrm{L}$ de $\mathrm{Mn}, 6,66 \mathrm{mg} / \mathrm{L}$ de $\mathrm{Na}, 0,06$ $\mathrm{mg} / \mathrm{L}$ de $\mathrm{Pb}$ e $0,31 \mathrm{mg} / \mathrm{L}$ de $\mathrm{Zn}), 12$ $(0,17 \mathrm{mg} / \mathrm{L}$ de $\mathrm{Cu}, 0,07 \mathrm{mg} / \mathrm{L}$ de $\mathrm{Fe}$, 25,90 mg/L de $\mathrm{Mn}, 4,64 \mathrm{mg} / \mathrm{L}$ de $\mathrm{Na}$, 0,13 mg/L de $\mathrm{Pb}$ e 0,22 mg/L de Zn), 13 $(0,40 \mathrm{mg} / \mathrm{L}$ de $\mathrm{Al}, 0,23 \mathrm{mg} / \mathrm{L}$ de $\mathrm{Fe}$, $0,68 \mathrm{mg} / \mathrm{L}$ de $\mathrm{Mn}, 5,70 \mathrm{mg} / \mathrm{L}$ de $\mathrm{Na}$, 0,05 mg/L de $\mathrm{Pb}$ e $0,42 \mathrm{mg} / \mathrm{L}$ de $\mathrm{Zn}), 14$ (3,30 mg/L de Ba e 0,04 mg/L de Pb) e 15 (3,46 mg/L de $\mathrm{Ba})$, em concordância com os laudos de classificação fornecidos pela empresa de destinação final de resíduos, não foram classificadas como classe I - perigosos por apresentarem os valores dos parâmetros analisados todos abaixo dos limites estabelecidos pela NBR 10004/04, então podendo ser classe II A- não inerte ou classe II B- inerte, dependendo do resultado encontrado na análise do extrato solubilizado (ABNT, 2004a).

No caso de comparação entre os limites estabelecidos pela NBR 10004/87, e a NBR 10004/04 verificou-se que a classe dos resíduos 11 , $12,13,14$ e 15 foi mantida, pois mesmo para parâmetros como bário, onde o limite passou de $100 \mathrm{mg} / \mathrm{L}$ para $70 \mathrm{mg} / \mathrm{L}$, e chumbo onde o limite passou de $5,0 \mathrm{mg} / \mathrm{L}$ para $1,0 \mathrm{mg} / \mathrm{L}$, os resultados encontrados foram bem inferiores.

$\mathrm{Na}$ Tabela 3 estão contidos os resultados dos parâmetros analisados pela unidade de disposição final e descritos no laudo de classificação de resíduos sólidos, os quais acompanharam as 18 amostras.

$\mathrm{Na}$ hipótese de que a lixiviação dos resíduos contidos na Tabela 3, ocorressem no meio ambiente, pode-se verificar pelos padrôes de lançamen- to de efluentes estabelecidos pelo Conselho Nacional do Meio Ambiente - CONAMA 357/2005, conforme a tabela 4, que apenas o $\mathrm{Cr}$ no resíduo $04 \mathrm{com} 2,0 \mathrm{mg} / \mathrm{L}$ e no resíduo $08 \mathrm{com}$ $0,6 \mathrm{mg} / \mathrm{L}$, o $\mathrm{Pb}$ no resíduo 05 com $0,53 \mathrm{mg} / \mathrm{L}$ e no resíduo $10 \mathrm{com}$ $0,6 \mathrm{mg} / \mathrm{L}$ e o $\mathrm{Zn}$ no resíduo $01 \mathrm{com}$ $53,30 \mathrm{mg} / \mathrm{L}$ estariam acima dos limites estabelecidos pela legislação.

Com o objetivo de também verificar a classificação dos resíduos, foram analisados alguns metais no extrato solubilizado. Na tabela 5 estão reunidos os resultados de cada amostra analisada nos Laboratórios do IAP.

Os resultados dos metais no extrato solubilizado evidenciaram que os resíduos 01, 02, 04, 05, 06, 07, 08, $09,10,11,12,13,14,15,17$ e 18 , são todos classificados como classe II A - não inertes por apresentarem pelo

Tabela 3 - Resultados dos parâmetros analisados no extrato lixiviado descritos no laudo de classificação de resíduos, elaborado pela unidade de disposição final

\begin{tabular}{|c|c|c|c|c|c|c|c|c|c|c|c|}
\hline \multirow[t]{2}{*}{ Referência } & \multirow[t]{2}{*}{ Classe } & \multicolumn{10}{|c|}{ Parâmetros $(\mathrm{mg} / \mathrm{L})$} \\
\hline & & $\mathrm{Al}$ & $\mathrm{Ba}$ & $\mathrm{Cd}$ & $\mathrm{Cu}$ & $\mathrm{Cr}$ & $\mathrm{Fe}$ & Fluoreto & $\mathrm{Na}$ & $\mathrm{Pb}$ & $\mathrm{Zn}$ \\
\hline $\begin{array}{l}\text { NBR10004/2004 } \\
\text { (Limite máximo) }\end{array}$ & IIA ou IIB & $\mathrm{NE}$ & 70,0 & 0,5 & $\mathrm{NE}$ & 5,0 & $\mathrm{NE}$ & 150 & $\mathrm{NE}$ & 1,0 & $\mathrm{NE}$ \\
\hline $\begin{array}{l}\text { NBR10004/1987 } \\
\text { (Limite máximo) }\end{array}$ & II ou III & $\mathrm{NE}$ & 100,0 & 0,5 & $\mathrm{NE}$ & 5,0 & $\mathrm{NE}$ & 150 & $\mathrm{NE}$ & 5,0 & $\mathrm{NE}$ \\
\hline $\begin{array}{l}\text { Limite de } \\
\text { Detecçáo }\end{array}$ & $\mathrm{NE}$ & $\mathrm{NE}$ & 0,4 & 0,01 & 0,1 & 0,1 & $\mathrm{NE}$ & 0,1 & $\mathrm{NE}$ & 0,2 & 0,008 \\
\hline Resíduo 01 & II ou III & NA & $<\mathrm{LD}$ & $<\mathrm{LD}$ & $<\mathrm{LD}$ & $<\mathrm{LD}$ & NA & 2,45 & NA & $<\mathrm{LD}$ & 53,30 \\
\hline Resíduo 02 & II ou III & NA & $<\mathrm{LD}$ & $<\mathrm{LD}$ & $<\mathrm{LD}$ & 0,41 & NA & $<\mathrm{LD}$ & NA & $<\mathrm{LD}$ & 0,42 \\
\hline Resíduo 03 & II ou III & NA & $<\mathrm{LD}$ & $<\mathrm{LD}$ & 0,15 & $<\mathrm{LD}$ & NA & 0,11 & NA & $<\mathrm{LD}$ & 0,14 \\
\hline Resíduo 04 & II ou III & NA & NA & NA & NA & 2,0 & NA & NA & NA & NA & NA \\
\hline Resíduo 05 & II ou III & NA & 1,0 & 0,027 & NA & $<\mathrm{LD}$ & NA & 0,18 & NA & 0,53 & NA \\
\hline Resíduo 06 & II ou III & NA & $<\mathrm{LD}$ & $<\mathrm{LD}$ & $<\mathrm{LD}$ & $<\mathrm{LD}$ & NA & 0,13 & NA & $<\mathrm{LD}$ & 0,17 \\
\hline Resíduo 07 & II ou III & NA & $<\mathrm{LD}$ & $<\mathrm{LD}$ & $<\mathrm{LD}$ & $<\mathrm{LD}$ & NA & 0,17 & NA & $<\mathrm{LD}$ & 0,10 \\
\hline Resíduo 08 & II ou III & NA & 1,0 & 0,018 & NA & 0,6 & NA & 0,80 & NA & 0,28 & NA \\
\hline Resíduo 09 & II ou III & NA & $<\mathrm{LD}$ & $<\mathrm{LD}$ & $<\mathrm{LD}$ & $<\mathrm{LD}$ & NA & 1,82 & NA & $<\mathrm{LD}$ & 0,40 \\
\hline Resíduo 10 & II ou III & NA & $<\mathrm{LD}$ & $<\mathrm{LD}$ & 0,4 & $<\mathrm{LD}$ & NA & 0,46 & NA & 0,60 & 0,009 \\
\hline Resíduo 11 & II ou III & NA & $<\mathrm{LD}$ & $<\mathrm{LD}$ & $<\mathrm{LD}$ & $<\mathrm{LD}$ & NA & 0,12 & NA & $<\mathrm{LD}$ & 0,46 \\
\hline Resíduo 12 & II ou III & NA & $<\mathrm{LD}$ & $<\mathrm{LD}$ & 0,16 & $<\mathrm{LD}$ & NA & NA & NA & $<\mathrm{LD}$ & 0,13 \\
\hline Resíduo 13 & II ou III & NA & $<\mathrm{LD}$ & $<\mathrm{LD}$ & $<\mathrm{LD}$ & $<\mathrm{LD}$ & NA & NA & NA & $<\mathrm{LD}$ & 0,20 \\
\hline Resíduo 14 & II ou III & NA & 2,90 & $<\mathrm{LD}$ & $<\mathrm{LD}$ & $<\mathrm{LD}$ & NA & NA & NA & $<\mathrm{LD}$ & NA \\
\hline Resíduo 15 & II ou III & NA & 2,86 & $<\mathrm{LD}$ & $<\mathrm{LD}$ & $<\mathrm{LD}$ & NA & NA & NA & $<\mathrm{LD}$ & NA \\
\hline Resíduo 16 & II ou III & NA & $<\mathrm{LD}$ & $<\mathrm{LD}$ & $<\mathrm{LD}$ & $<\mathrm{LD}$ & NA & 0,92 & NA & $<\mathrm{LD}$ & 0,34 \\
\hline Resíduo 17 & II ou III & NA & $<\mathrm{LD}$ & $<\mathrm{LD}$ & $<\mathrm{LD}$ & $<\mathrm{LD}$ & NA & 2,11 & NA & $<\mathrm{LD}$ & 0,11 \\
\hline Resíduo 18 & II ou III & NA & $<\mathrm{LD}$ & $<\mathrm{LD}$ & $<\mathrm{LD}$ & $<\mathrm{LD}$ & NA & 0,53 & NA & $<\mathrm{LD}$ & 0,03 \\
\hline
\end{tabular}




\begin{tabular}{cc}
$\begin{array}{c}\text { Tabela } 4 \text { - Teores máximos de algumas substâncias para lançamento de efluentes } \\
\text { estabelecidos pela legislação nacional - CONAMA 357/2005 }\end{array}$ \\
\hline Substância & Lançamento $(\mathrm{mg} / \mathrm{L})$ \\
\hline Bário $(\mathrm{Ba})$ & 5,0 \\
Cádmio $(\mathrm{Cd})$ & 0,2 \\
Chumbo $(\mathrm{Pb})$ & 0,5 \\
Cobre $(\mathrm{Cu})$ & 1,0 \\
Cromo total $(\mathrm{Cr})$ & 0,5 \\
Ferro $(\mathrm{Fe})$ & 15,0 \\
Fluoretos & 10,0 \\
Manganês $(\mathrm{Mn})$ & 1,0 \\
Zinco $(\mathrm{Zn})$ & 5,0
\end{tabular}

Fonte: Resolução CONAMA 357/2005 - Art. 34

Tabela 5 - Resultados das análises de metais no extrato solubilizado

\begin{tabular}{|c|c|c|c|c|c|c|c|c|c|c|c|}
\hline \multirow[t]{2}{*}{ Referência } & \multirow[t]{2}{*}{ Classe } & \multicolumn{10}{|c|}{ Metais $(\mathrm{mg} / \mathrm{L})$} \\
\hline & & $\mathrm{Al}$ & $\mathrm{Ba}$ & $\mathrm{Cd}$ & $\mathrm{Cu}$ & $\mathrm{Cr}$ & $\mathrm{Fe}$ & $\mathrm{Mn}$ & $\mathrm{Na}$ & $\mathrm{Pb}$ & $\mathrm{Zn}$ \\
\hline $\begin{array}{l}\text { NBR10004 /2004 } \\
\text { (Limite máximo) }\end{array}$ & IIA ou IIB & 0,20 & 0,7 & 0,005 & 2,0 & 0,05 & 0,30 & 0,10 & 200,0 & 0,01 & 5,0 \\
\hline $\begin{array}{c}\text { NBR10004 /1987 } \\
\text { (Limite máximo) }\end{array}$ & II ou III & 0,20 & 1,0 & 0,005 & 1,0 & 0,05 & 0,30 & 0,10 & 200,0 & 0,05 & 5,0 \\
\hline $\begin{array}{l}\text { Limite de } \\
\text { Detecção }\end{array}$ & $\mathrm{NE}$ & 0,40 & 0,60 & 0,01 & 0,03 & 0,10 & 0,06 & 0,03 & 0,01 & 0,04 & 0,10 \\
\hline Resíduo 01 & II & 0,53 & NA & NA & NA & NA & $<\mathrm{LD}$ & 19,15 & 378,0 & NA & 61,25 \\
\hline Resíduo 02 & II & $<\mathrm{LD}$ & NA & NA & NA & 2,22 & 0,80 & 0,06 & 144,0 & NA & $<\mathrm{LD}$ \\
\hline Resíduo 03 & III & NA & NA & NA & 0,27 & NA & 0,06 & $<\mathrm{LD}$ & 69,40 & NA & $<\mathrm{LD}$ \\
\hline Resíduo 04 & II & $<\mathrm{LD}$ & NA & NA & NA & NA & 0,06 & 1,24 & 1125,0 & NA & 0,54 \\
\hline Resíduo 05 & II & $<\mathrm{LD}$ & NA & NA & $<\mathrm{LD}$ & $<\mathrm{LD}$ & 5,90 & $<\mathrm{LD}$ & 117,85 & $<\mathrm{LD}$ & 0,17 \\
\hline Resíduo 06 & II & $<\mathrm{LD}$ & NA & NA & $<\mathrm{LD}$ & $<\mathrm{LD}$ & $<\mathrm{LD}$ & $<\mathrm{LD}$ & 273,4 & $<\mathrm{LD}$ & $<\mathrm{LD}$ \\
\hline Resíduo 07 & II & $<\mathrm{LD}$ & NA & NA & $<\mathrm{LD}$ & $<\mathrm{LD}$ & 0,31 & 0,57 & 9,78 & $<\mathrm{LD}$ & $<\mathrm{LD}$ \\
\hline Resíduo 08 & II & 0,53 & $<\mathrm{LD}$ & NA & $<\mathrm{LD}$ & $<\mathrm{LD}$ & 0,37 & NA & 24,71 & $<\mathrm{LD}$ & $<\mathrm{LD}$ \\
\hline Resíduo 09 & II & $<\mathrm{LD}$ & NA & NA & $<\mathrm{LD}$ & $<\mathrm{LD}$ & 0,11 & 0,72 & 7,99 & $<\mathrm{LD}$ & $<\mathrm{LD}$ \\
\hline Resíduo 10 & II & 16,00 & NA & NA & 0,09 & $<\mathrm{LD}$ & 0,17 & $<\mathrm{LD}$ & 22,74 & $<\mathrm{LD}$ & $<\mathrm{LD}$ \\
\hline Resíduo 11 & II & $<\mathrm{LD}$ & NA & NA & $<\mathrm{LD}$ & $<\mathrm{LD}$ & 0,62 & 0,08 & 25,56 & $<\mathrm{LD}$ & 1,29 \\
\hline Resíduo 12 & II & $<\mathrm{LD}$ & NA & NA & NA & $<\mathrm{LD}$ & 0,36 & $<\mathrm{LD}$ & 10,63 & $<\mathrm{LD}$ & $<\mathrm{LD}$ \\
\hline Resíduo 13 & II & $<\mathrm{LD}$ & NA & NA & NA & $<\mathrm{LD}$ & 1,54 & 0,10 & 18,62 & $<\mathrm{LD}$ & $<\mathrm{LD}$ \\
\hline Resíduo 14 & II & $<\mathrm{LD}$ & 2,02 & $<\mathrm{LD}$ & $<\mathrm{LD}$ & $<\mathrm{LD}$ & $<\mathrm{LD}$ & $<\mathrm{LD}$ & 4,85 & $<\mathrm{LD}$ & $<\mathrm{LD}$ \\
\hline Resíduo 15 & II & $<\mathrm{LD}$ & 1,33 & $<\mathrm{LD}$ & $<\mathrm{LD}$ & $<\mathrm{LD}$ & $<\mathrm{LD}$ & $<\mathrm{LD}$ & 4,39 & $<\mathrm{LD}$ & $<\mathrm{LD}$ \\
\hline Resíduo 16 & III & NA & NA & NA & $<\mathrm{LD}$ & NA & 0,16 & 0,04 & 52,95 & NA & $<\mathrm{LD}$ \\
\hline Resíduo 17 & II & $<\mathrm{LD}$ & NA & NA & 0,03 & $<\mathrm{LD}$ & 10,05 & 0,19 & 22,59 & $<\mathrm{LD}$ & 0,16 \\
\hline Resíduo 18 & II & NA & NA & NA & NA & NA & 0,54 & NA & NA & NA & $<\mathrm{LD}$ \\
\hline
\end{tabular}


menos um dos parâmetros analisados com valor acima dos limites estabelecidos pela NBR 10004/04 (ABNT, 2004a). Já os resíduos 03 e 16, foram classificados como classe II B - inertes por apresentarem todos os parâmetros com valores abaixo dos limites estabelecidos pela NBR 10004/04.

Comparando os limites estabelecidos pela ABNT (1987a), e a ABNT (2004a), observa-se que dos parâmetros analisados, o bário passou de $1,0 \mathrm{mg} / \mathrm{L}$ para $0,7 \mathrm{mg} / \mathrm{L}$, o cobre passou de $1,0 \mathrm{mg} / \mathrm{L}$ para $2,0 \mathrm{mg} / \mathrm{L}$ e o chumbo passou de $0,05 \mathrm{mg} / \mathrm{L}$ para $0,01 \mathrm{mg} / \mathrm{L}$. De acordo com esta consideração, a alteração destes limites não significou mudança de classe para os resíduos 01 , 02, 04, 05, 06, 07, 08, 09, 10, 11, 12, $13,14,15,17$ e 18, classificados como classe II $\mathrm{A}$, pois estes não dependeram das concentrações detectadas de bário, cobre e chumbo para determinar a classe. No entanto, para os resíduos 03 e 16, os quais foram classificados como classe II $\mathrm{B}$, o chumbo poderia ser o parâmetro decisivo para a confirmação ou não da classe estabelecida para estes resíduos, pois, levando-se em consideração esta possibilidade, o chumbo deveria ser analisado com limite de quantificação menor que $0,04 \mathrm{mg} / \mathrm{L}$ pelo fato de que o limite máximo passou de $0,05 \mathrm{mg} / \mathrm{L}$ para $0,01 \mathrm{mg} / \mathrm{L}$, sendo este um valor bem menor que o limite detectado pela análise.

$\mathrm{Na}$ Tabela 6 estão dispostos os resultados dos parâmetros analisados no extrato solubilizado contidos nos laudos de classificação de resíduos sólidos da unidade de destinação final.
Quando é realizada a comparação das classes obtidas na Tabela $5 \mathrm{com}$ as fornecidas pelos laudos de classificação da unidade de destinação final de resíduos, descritos na Tabela 6, observa-se a compatibilidade quase absoluta entre os resultados, salvo três resíduos onde a classe difere. Provavelmente, esta diferença é devida a não homogeneidade da amostra, sendo que esta pode ter sido coletada em um determinado ponto e enviado para a classificação no laboratório da unidade de destinação final, e em outro ponto para a classificação realizada pelo laboratório do IAP. Salienta-se também que a qualidade dos resultados pode ser influenciada diretamente pelos procedimentos de coleta adotados, sendo aceitável esta incompatibilidade de classificação das amostras 03 , 08 e 16 com seus laudos.

Tabela 6 - Resultados dos metais analisados no extrato solubilizado descritos no laudo de classificação de resíduos sólidos, elaborado pela unidade de destinação final

\begin{tabular}{|c|c|c|c|c|c|c|c|c|c|c|c|}
\hline \multirow[t]{2}{*}{ Referência } & \multirow[t]{2}{*}{ Classe } & \multicolumn{10}{|c|}{ Metais $(\mathrm{mg} / \mathrm{L})$} \\
\hline & & $\mathrm{Al}$ & $\mathrm{Ba}$ & $\mathrm{Cd}$ & $\mathrm{Cu}$ & $\mathrm{Cr}$ & $\mathrm{Fe}$ & $\mathrm{Mn}$ & $\mathrm{Na}$ & $\mathrm{Pb}$ & $\mathrm{Zn}$ \\
\hline $\begin{array}{l}\text { NBR10004 /2004 } \\
\text { (Limite } \\
\text { máximo) }\end{array}$ & IIA ou IIB & 0,20 & 0,7 & 0,005 & 2,0 & 0,05 & 0,30 & 0,10 & 200,0 & 0,01 & 5,0 \\
\hline $\begin{array}{l}\text { NBR10004 /1987 } \\
\text { (Limite } \\
\text { máximo) }\end{array}$ & II ou III & 0,20 & 1,0 & 0,005 & 1,0 & 0,05 & 0,30 & 0,10 & 200,0 & 0,05 & 5,0 \\
\hline $\begin{array}{l}\text { Limite de } \\
\text { Detecçãa }\end{array}$ & $\mathrm{NE}$ & 0,15 & 0,1 & 0,005 & 0,025 & 0,025 & 0,05 & 0,02 & 0,05 & 0,05 & 0,002 \\
\hline Resíduo 01 & II & 0,950 & $<\mathrm{lD}$ & $<\mathrm{lD}$ & $<\mathrm{lD}$ & $<\mathrm{lD}$ & 0,71 & 11,28 & 304,0 & $<\mathrm{lD}$ & 75,20 \\
\hline Resíduo 02 & II & 4,31 & $<\mathrm{lD}$ & $<\mathrm{lD}$ & $<\mathrm{lD}$ & 0,91 & 6,56 & 0,73 & 915,0 & $<\mathrm{lD}$ & 0,30 \\
\hline Resíduo 03 & II & $<\mathrm{lD}$ & $<\mathrm{lD}$ & $<\mathrm{lD}$ & 0,04 & $<\mathrm{lD}$ & 2,22 & 0,37 & 1,41 & $<\mathrm{lD}$ & 0,15 \\
\hline Resíduo 04 & II & NA & NA & NA & NA & 2,5 & NA & NA & NA & NA & NA \\
\hline Resíduo 05 & II & 0,36 & $<\mathrm{LD}$ & 0,006 & $<\mathrm{LD}$ & $<\mathrm{LD}$ & 0,18 & 0,09 & 25,0 & 0,42 & 0,01 \\
\hline Resíduo 06 & II & $<\mathrm{lD}$ & $<\mathrm{lD}$ & $<\mathrm{lD}$ & $<\mathrm{lD}$ & $<\mathrm{lD}$ & 0,33 & $<\mathrm{lD}$ & 169,0 & $<\mathrm{lD}$ & 0,02 \\
\hline Resíduo 07 & II & $<1 \mathrm{D}$ & $<\mathrm{lD}$ & $<\mathrm{lD}$ & $<\mathrm{lD}$ & $<\mathrm{lD}$ & 432,4 & 0,12 & 34,8 & $<\mathrm{lD}$ & 0,03 \\
\hline Resíduo 08 & III & 0,17 & $<\mathrm{LD}$ & $<\mathrm{LD}$ & $<\mathrm{LD}$ & $<\mathrm{lD}$ & 0,1 & 0,08 & 17,0 & $<\mathrm{LD}$ & 0,01 \\
\hline Resíduo 09 & II & 0,28 & $<\mathrm{lD}$ & $<\mathrm{lD}$ & $<\mathrm{lD}$ & $<\mathrm{lD}$ & 0,54 & 1,42 & 5,94 & $<\mathrm{lD}$ & 0,05 \\
\hline Resíduo 10 & II & 1,61 & $<\mathrm{lD}$ & $<\mathrm{lD}$ & $<\mathrm{lD}$ & $<\mathrm{lD}$ & 0,06 & $<\mathrm{lD}$ & 18,20 & $<\mathrm{lD}$ & 0,009 \\
\hline Resíduo 11 & II & 0,20 & $<\mathrm{lD}$ & $<\mathrm{lD}$ & $<\mathrm{lD}$ & $<\mathrm{lD}$ & 1,00 & 0,18 & 51,0 & $<\mathrm{lD}$ & 0,12 \\
\hline Resíduo 12 & II & $<\mathrm{LD}$ & NA & NA & NA & $<\mathrm{lD}$ & 0,48 & $<\mathrm{LD}$ & 17,50 & $<\mathrm{LD}$ & $<\mathrm{LD}$ \\
\hline Resíduo 13 & II & $<\mathrm{LD}$ & NA & NA & NA & $<\mathrm{lD}$ & 1,36 & $<\mathrm{LD}$ & 28,60 & $<\mathrm{LD}$ & $<\mathrm{LD}$ \\
\hline Resíduo 14 & II & $<\mathrm{LD}$ & 1,98 & NA & $<\mathrm{LD}$ & $<\mathrm{lD}$ & $<\mathrm{LD}$ & $<\mathrm{LD}$ & 3,84 & $<\mathrm{LD}$ & $<\mathrm{LD}$ \\
\hline Resíduo 15 & II & $<\mathrm{LD}$ & 1,50 & NA & $<\mathrm{LD}$ & $<\mathrm{lD}$ & $<\mathrm{LD}$ & $<\mathrm{LD}$ & 4,36 & $<\mathrm{LD}$ & $<\mathrm{LD}$ \\
\hline Resíduo 16 & II & $<1 \mathrm{D}$ & $<\mathrm{lD}$ & 0,11 & 0,027 & $<\mathrm{lD}$ & 1,79 & $<\mathrm{lD}$ & 8,03 & $<\mathrm{lD}$ & 0,29 \\
\hline Resíduo 17 & II & $<\mathrm{lD}$ & $<\mathrm{lD}$ & $<\mathrm{lD}$ & $<\mathrm{lD}$ & $<\mathrm{lD}$ & 8,84 & 0,15 & 4,80 & $<\mathrm{lD}$ & 0,06 \\
\hline Resíduo 18 & II & NA & NA & NA & NA & NA & 0,53 & NA & NA & NA & 0,05 \\
\hline
\end{tabular}


Considerando a possibilidade de que a solubilização dos resíduos relacionados na tabela 6 , ocorressem no meio ambiente, pode-se verificar pelos padróes de lançamento de efluentes estabelecido pela Resolução CONAMA 357/05, já descritos na Tabela 4, que o extrato solubilizado do resíduo $01 \mathrm{com}$ $11,28 \mathrm{mg} / \mathrm{L}$ de $\mathrm{Mn}$ e com 75,20 mg/L de $\mathrm{Zn}$, do resíduo $02 \mathrm{com} 0,91 \mathrm{mg} / \mathrm{L}$ de $\mathrm{Cr}$, do resíduo $04 \mathrm{com} 2,5 \mathrm{mg} / \mathrm{L}$ de $\mathrm{Cr}$, do resíduo 07 com 432,4 mg/L de Fe, e também do resíduo 09 com $1,42 \mathrm{mg} / \mathrm{L}$ de $\mathrm{Mn}$, estariam com valores acima dos limites para lançamento.

Os testes de toxicidade foram aplicados no extrato solubilizado das dezoito amostras de resíduos, como também em quatro coletas de drenados dos aterros classe IIA e classe IIB. Pode-se dizer que a diversidade na tipologia das amostras foi atingida, pois desejava-se ter dados de toxicidade de vários ramos diferentes do setor industrial.

$\mathrm{Na}$ Tabela 7 são apresentados os resultados das análises de toxicidade.

De acordo com esta análise podese verificar que todas as amostras apresentaram toxicidade para as bactérias, no entanto, para os microcrustáceos apenas a amostra 06 não foi considerada tóxica por apresentar FT igual a 1. Quando o resultado de toxicidade apresentar valor maior que 1 a amostra apresenta toxicidade aguda, mas, se este valor for igual a 1 a amostra não é tóxica, pois não precisou ser diluída para não ocorrer efeito tóxico aos organismos (Knie e Lopes, 2004).

Verifica-se, também, que algumas amostras causam o efeito tóxico maior para as bactérias como os resíduos 02 , 03, 06, 07, 08, 09, 11, 13, 17 e 18 . Outras, como os resíduos $01,10,12$, 14, 15 e 16, para os microcrustáceos e outras como os resíduos 04 e 05 apresentam a mesma toxicidade a ambos os organismos. Isto demonstra a diferença de sensibilidade entre os organismos bioindicadores de toxicidade.

Os dados descritos na Tabela 7 foram analisados com base na Portaria $N^{\circ}$ 017/02 da Fundação do Meio Ambiente de Santa Catarina - FATMA, órgão fiscalizador do Estado de Santa Catarina, onde esta estabelece limites máximos de toxicidade, utilizando dois organismos bioindicadores de toxicidade (D. magna e $V$. fischeri), para efluentes de diferentes origens, divididos em categorias.

De acordo com a Portaria $\mathrm{N}^{\circ}$ 017/02 da FATMA, somente o extrato solubilizado dos resíduos 04 , 14 e 15, dos dezoito analisados, estão dentro dos limites de toxicidade para lançamento. Observa-se também que o maior efeito tóxico, para ambos os organismos testados, ocorreu no resíduo 11 , originado no processo de fabricação de resina, com um fator de toxicidade de 256 para a bactéria luminescente, onde o limite é 4, e com um fator de toxicidade de 128 para o microcrustáceo, onde o limite é 2 . Isto se deve a presença de uma quantidade maior de substâncias tóxicas na amostra e a interação entre elas (Nieweglowski e Silva, 1999). Cabe ressaltar que a sensibilidade mais elevada se deu para as bactérias, comparando com a sensibilidade do outro organismo teste.

$\mathrm{Na}$ Tabela 8 estão apresentados os resultados dos testes de toxicidade aguda realizados com o drenado dos aterros classe IIA e classe IIB.

Constatou-se através dos ensaios com os drenados dos aterros que o efeito tóxico do somatório dos resíduos, onde encontram-se diferentes substâncias, é muito maior que a toxicidade individual de cada resíduo. Os efluentes se mostram bastante tóxicos para ambos os organismos testados, no entanto, as bactérias apresentaram mais

Tabela 7 - Resultados dos testes de toxicidade aguda no extrato solubilizado

\begin{tabular}{|c|c|c|c|}
\hline \multirow[t]{2}{*}{ Amostra } & \multicolumn{3}{|c|}{ Fator de toxicidade } \\
\hline & Bactéria & Vibrio fischeri & Microcrustáceo Daphnia magna \\
\hline Resíduo 01 & & 16 & 128 \\
\hline Resíduo 02 & & 128 & 4 \\
\hline Resíduo 03 & & 32 & 16 \\
\hline Resíduo 04 & & 2 & 2 \\
\hline Resíduo 05 & & 32 & 32 \\
\hline Resíduo 06 & & 8 & 1 \\
\hline Resíduo 07 & & 8 & 2 \\
\hline Resíduo 08 & & 64 & 4 \\
\hline Resíduo 09 & & 16 & 2 \\
\hline Resíduo 10 & & 64 & 128 \\
\hline Resíduo 11 & & 256 & 128 \\
\hline Resíduo 12 & & 4 & 8 \\
\hline Resíduo 13 & & 16 & 4 \\
\hline Resíduo 14 & & 2 & 4 \\
\hline Resíduo 15 & & 2 & 4 \\
\hline Resíduo 16 & & 2 & 8 \\
\hline Resíduo 17 & & 32 & 8 \\
\hline Resíduo 18 & & 32 & 4 \\
\hline
\end{tabular}

Tabela 8 - Resultados dos testes de toxicidade no drenado do aterro classe II A e classe II B

\begin{tabular}{cccc}
\hline Data da coleta & Condiçóes do tempo & \multicolumn{2}{c}{ Fator de toxicidade } \\
& & $\begin{array}{c}\text { Bactéria } \\
\text { Vibrio fischeri }\end{array}$ & $\begin{array}{c}\text { Microcrustáceo } \\
\text { Daphnia magna }\end{array}$ \\
\hline $13 / 09 / 04$ & Com chuva & 256 & 128 \\
$16 / 09 / 04$ & Sem chuva & 256 & 64 \\
$20 / 09 / 04$ & Com sol & 256 & 64 \\
$24 / 09 / 04$ & Dia nublado & 256 & 64 \\
\hline
\end{tabular}


sensibilidade para todas as amostras, as quais foram coletadas em datas e condições do tempo variadas.

Os drenados dos aterros classe II A - não inertes e classe II B - inertes, se comparados com a Portaria 017/02 da FATMA, se enquadram nas categorias de efluentes não definidas, onde ficam estabelecidos limites de toxicidade, descritos em um parágrafo único, de FT igual a 8 para $D$. magna e para $V$. fischeri. Estes drenados apresentaram para D. magna valores de FT igual a 128 , quando coletado em dia chuvoso, e FT igual a 64 nas outras três coletas quando não chovia. Já, os valores encontrados para $V$. fischeri foram de FT igual a 256, independente da data e condições do tempo da coleta. Comparando estes valores com os limites estabelecidos pela FATMA (2002), verifica-se que todos se encontram bem superiores.

Com os resultados de toxicidade chegando à um FT igual a 256, tanto no extrato solubilizado dos resíduos quanto no drenado dos aterros, verifica-se a necessidade de estabelecer limites de toxicidade para lançamento de efluentes. Ainda, como os drenados dos aterros apresentaram-se bastante tóxicos, conforme verificado pela tabela 8 , ocorre a necessidade da aplicação de tratamentos para a redução da toxicidade. O grande problema seria a tratabilidade destes drenados, pois segundo Pawlowsky (1994) é extremamente complicado utilizar processos biológicos diretamente para estes despejos, surgindo a necessidade de uma difícil e cara detoxificação prévia, como tratamentos físico-químicos por coagulação/floculação, carvão ativado, processos oxidativos, processos por membranas, entre outros, para reduzir a toxicidade aguda a limites aceitáveis.

Este procedimento faria com que o custo de disposição final em aterros aumentasse consideravelmente. Assim, o caminho mais adequado seria minimizar a geração de drenados muito tóxicos ou limitar a toxicidade do resíduo original. Uma das alternativas seria estabelecer padrōes de toxicidade para o extrato solubilizado dos resíduos, evitando com isto que resíduos altamente tóxicos fossem dispostos no aterro classe II A ou II B, mas sim, no aterro classe I. Assim, diminuiria a geração e o volume de drenados muito tóxicos a serem submetidos à detoxificação, conseqüentemente baixando o custo da disposição.

\section{CONCLUSÕES}

Os resultados obtidos com testes de toxicidade aguda permitiram concluir que foi possível conhecer o nível tóxico do extrato solubilizado dos resíduos de diversos ramos industriais, conforme apresentado na tabela 7 , classificados como classe II A e II B, onde o fator de toxicidade variou de 1 a 128 para D. magna e de 2 a 256 para V. fischeri.

Os resultados para a toxicidade encontrados no aterro de resíduos classe IIA e IIB demonstraram valores iguais aos mais tóxicos analisados individualmente. Deve haver uma interação entre os resíduos no aterro, fazendo com que predominem os de maior fator de toxicidade, por ações sinérgicas e antagônicas.

Assim, deverá ser estabelecido limites de toxicidade para cada extrato solubilizado individual, de acordo com seu ramo industrial, como também para os drenados dos aterros de resíduos como se fossem substâncias novas e únicas em sua composição.

Também, ficou demonstrado através das análises de lixiviação (Tabela 3) dos resíduos 02, 03, 06, 07, 09, 11, 12, $13,14,15,16,17,18$, e de solubilização (tabela 6) dos resíduos 03, 05, 06, 08, $10,11,12,13,14,15,16,17,18$, que efluentes líquidos que atendem aos padrões de lançamento, não estão isentos de causar efeito tóxico ao corpo receptor, conforme apresentou a tabela 7 para os resíduos 01, 02, 03, 05, 06, 07, 08, 09, $10,11,12,13,16,17$ e 18.

As informações obtidas pelo estudo de extratos solubilizados usando análises de metais e ensaios de toxicidade reforçam a idéia de que esses devem ser utilizados em conjunto para garantir o nível de segurança quanto ao padrão de qualidade das águas, disposto na Resolução CONAMA 357/05. Somente o extrato solubilizado dos resíduos 04,14 e 15 , estão dentro dos limites de toxicidade para lançamento, para os dois organismos indicadores de toxicidade (D. magna e $V$. fischeri), segundo a Portaria da FATMA. Como a legislação exige controle e, através dos dados de FT apresentados na tabela 8 , constatou-se a existência de toxicidade bastante elevada no drenado do aterro, ocorrendo a necessidade de se detoxificar e tratar.

Em termos de sensibilidade, os dois organismos teste utilizados se mos- traram praticamente similares, sendo que a bactéria Vibrio fischeri foi mais sensível para 10 amostras de extrato solubilizado $(02,03,06,07,08,09,11$, 13,17 e 18) e para todas as amostras de drenados, e a Daphnia magna para 6 amostras de extrato solubilizados $(01,10,12,14,15$ e 16$)$. Nos extratos solubilizados dos resíduos $04(\mathrm{FT}=2)$ e 05 ( $\mathrm{FT}=32)$, a sensibilidade foi a mesma para os dois organismos teste.

\section{REFERÊNCIAS}

ABNT - ASSOCIAÇÃO BRASILEIRA DE NORMAS TÉCNICAS. NBR 10004: Classificação de Resíduos. Rio de Janeiro: p. 71. 2004 a.

ABNT - ASSOCIAÇÃO BRASILEIRA DE NORMAS TÉCNICÁS. NBR 10005: Lixiviação de Resíduos - Procedimento. Rio de Janeiro: p. $7.1987 \mathrm{~b}$

ABNT - ASSOCIAÇÃO BRASILEIRA DE NORMAS TÉCNICAS. NBR 10005: Lixiviação de Resíduos - Procedimento. Rio de Janeiro: p. 16. $2004 \mathrm{~b}$.

ABNT - ASSOCIAÇÃO BRASILEIRA DE NORMAS TÉCNICÁS. NBR 10006: Solubilização de Resíduos - Procedimento. Rio de Janeiro: p. 2. 1987 c.

ABNT - ASSOCIAÇÃO BRASILEIRA DE NORMAS TÉCNICAS. NBR 10006: Solubilização de Resíduos - Procedimento. Rio de Janeiro: p. 3. 2004 c.

ABNT - ASSOCIAÇÃO BRASILEIRA DE NORMAS TÉCNICAS. NBR 12713: Ecotoxicologia Aquática - Toxicidade Aguda - Método de Ensaio com daphnia spp (Cladocera, Crustácea). Rio de Janeiro: p. 17. 2004e.

APHA. Standard methods for the Examination of Water ad Wastewater. 20. ed. USA: American Public Health Association, 1998.

CARDOSO, L.; LUCA, S. J. Toxidez de efluentes municipais biologicamente tratados e desinfetados com hipoclorito e ferrato de sódio. In: Revista Engenharia Sanitária e Ambiental, v.9, n. 1, p. 26-29, 2004.

CETESB - COMPANHIA DE TECNOLOGIA DE SANEAMENTO AMBIENTAL. Procedimentos para utilização de testes de toxicidade no controle de efluentes líquidos. São Paulo: CETESB, p. 17. $1990 \mathrm{~b}$.

CONAMA 357/05. Resolução CONAMA $\mathrm{n}^{\circ} 357$, de 17 de março de 2005. Dispōe sobre a classificação dos corpos de água e diretrizes ambientais para seu enquadramento, bem como estabelece as condiçōes e padrōes de lançamento de efluentes, e dá outras providências. Brasília, 2005.

FATMA - FUNDAÇÃO DO MEIO AMBIENTE DE SANTA CATARINA. Relevância de parâmetros de qualidade das águas aplicados à águas correntes. Parte I. Florianópolis: FATMA/GTZ, p. 108. 1999.

FATMA - FUNDAÇÃO DO MEIO AMBIENTE DE SANTA CATARINA. Portaria ${ }^{\circ}$ 017/02 de 18 de abril de 2002. Limites Máximos de Toxicidade Aguda para Efluentes de Diferentes Origens. 2002. 
IAP - INSTITUTO AMBIENTAL DO PARANÁ. Manual de métodos para avaliação de toxicidade. Curitiba: IAP, p. 101. 1997.

ISO - INTERNATIONAL ORGANIZATION FOR STANDARDIZATION. Water QualityDetermination of the Mobility of Daphnia magna Straus (Cladocera, Cruatácea). ISO 6341. Geneve, 1993b.

JACOMINO, V. M. F. et al. Controle Ambiental das Indistrias de Ferro-Gussa em Altos-Fornos a Carvão Vegetal. Belo Horizonte: Projeto Minas Ambiente, p. 181-197. 2002.

KNIE, J. L. W.; LOPES, E. W. B. Testes ecotoxicológicos: métodos, técnicas e aplicaçōes. Florianópolis: FATMA / GTZ, p. 289, 2004

NIEWEGLOWSKI, A. M. A.; SILVA, E. M. de F. M. Importância dos Parâmetros Ecotoxicológicos em Estudos Ambientais. In: MANUAL DE AVALIACÃO DE IMPACTOS AMBIENTAIS, Curitiba:SUREHMA, p. 6. 1999.

PAWLOWSKY, U. Tratabilidade de Efluentes de Fabricação de Herbicidas. Tese para Concurso Público de Professor Titular de Engenharia Ambiental - Setor de Tecnologia, Universidade Federal do Paraná. Curitiba, 318f. 1994.

ZAGATTO, P. A. et al. Avaliação de toxicidade em sistema de tratamento biológico de afluentes líquidos. In: Revista SABESP, n. 166, p. 1-6, 1992.

ZULAUF, W. E. Residuos Sólidos Industriais. In: SEMINÁRIO DE UTILIDADES, 2., 1977, São Paulo. CETESB-ABLP. p. 7. 1977

Endereço para correspondência:

Nébora Liz Vendramin Brasil

Rodrigues

Quartel General / $5^{a}$ Região

Militar

Rua 3 I de Março, s/no

Pinheirinho

81 I50-900 Curitiba - PR - Brasil

80230-000 Curitiba - PR - Brasil

Tel: (4I) 9 I 84-7494

E-mail: neborarodrigues@ig.com.br 